\title{
Dual roles of Drosophila p53 in cell death and cell differentiation
}

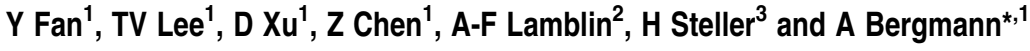

The mammalian $\mathrm{p} 53$ family consists of $\mathrm{p} 53, \mathrm{p} 63$ and $\mathrm{p} 73$. Whereas $\mathrm{p} 53$ accounts for tumor suppression through cell-cycle arrest and apoptosis, the functions of p63 and p73 are more diverse and also include control of cell differentiation. The Drosophila genome contains only one p53 homolog, Dp53. Previous work has established that Drosophila p53 (Dp53) induces apoptosis, but not cell-cycle arrest. In this study, using the developing eye as a model, we show that Dp53-induced apoptosis is primarily dependent on the pro-apoptotic gene, head involution defective (hid), but not reaper (rpr), and occurs through the canonical apoptosis pathway. Importantly, similar to p63 and p73, expression of Dp53 also inhibits cellular differentiation of photoreceptor neurons and cone cells in the eye independently of its apoptotic function. Intriguingly, expression of the human cell-cycle inhibitor p21 or its Drosophila homolog dacapo (dap) can suppress both Dp53-induced cell death and differentiation defects in Drosophila eyes. These findings provide new insights into the pathways activated by Dp53 and reveal that Dp53 incorporates functions of multiple p53 family members.

Cell Death and Differentiation (2010) 17, 912-921; doi:10.1038/cdd.2009.182; published online 4 December 2009

p53 is a well-known tumor suppressor. Depending on cell type and cellular context, activation of p53 can trigger multiple cellular responses, including cell-cycle arrest and apoptosis (reviewed in Vousden and Prives ${ }^{1}$ ). Although p53 functions through several mechanisms, it has been best characterized as a transcription factor that activates target genes including the cyclin-dependent kinase (CDK) inhibitor p21 and the pro-apoptotic genes Puma and Noxa. ${ }^{1}$ In addition, p63 and $p 73$, two $p 53$ paralogs, have been identified in vertebrates (reviewed in Pietsch et al. $^{2}$ and Stiewe ${ }^{3}$ ). They can not only induce apoptosis, but also have additional functions because $p 63^{-1-}$ and $p 73^{-1-}$ knockout mice show clear developmental defects, in contrast to $p 53^{-1-}$-null mice that are viable and develop normally..$^{2,3}$ The analysis of the $p 63^{-1-}$ phenotype revealed that $p 63$ is required for epithelial stem cell maintenance. In the absence of p63, these stem cells undergo terminal differentiation and do not remain to sustain the epidermis. ${ }^{4} p 63^{-1-}$ mice die shortly after birth. The $p 73^{-1-}$ phenotype is more complex, and also includes differentiation defects of certain populations of neurons in the brain. Further complicating is the observation that there are antagonistic p53 family members that are produced from additional intronic promoters generating $\mathrm{N}$-terminally truncated $(\Delta \mathrm{N})$ isoforms (reviewed by Stiewe ${ }^{3}$ and Murray-Zmijewski et al. ${ }^{4}$ ). The $\Delta \mathrm{N}$ isoforms can bind with the full-length transactivating (TA) isoforms of p53, p63 and p73, and antagonize their functions. ${ }^{3,4}$ Thus, this complexity makes it very difficult to dissect the functional mechanisms of the p53 family members in vertebrates. It is therefore attractive to examine the ancestral function of p53 orthologs in invertebrates such as Drosophila.

The Drosophila genome contains a single p53 family member, referred to as Drosophila p53 (Dp53). ${ }^{5-7}$ Similar to mammalian p53, Dp53-null mutant flies are viable and fertile, and with the exception of an apoptotic defect of primordial germ cells, they have no obvious developmental defects. ${ }^{8,9}$ In contrast to mammalian p53, Dp53 appears unable to induce radiation-induced cell-cycle arrest. ${ }^{5,6,8}$ Similarly, mammalian cells lacking p63 and p73 are also unable to induce DNA damage-induced cell-cycle arrest. ${ }^{10}$ Consequently, Dp53 and various forms of irradiation do not induce the expression of the Drosophila p21 homolog, dacapo (dap). ${ }^{8,11}$

Importantly, the pro-apoptotic function of p53 is well conserved in Drosophila. In response to apoptotic stimuli, the pro-apoptotic genes reaper ( $r p r)$, head involution defective (hid) and grim are both necessary and sufficient to induce apoptosis through inhibition of the caspase inhibitor Diap1, which subsequently leads to activation of the initiator caspase, Drosophila Nedd2-like caspase (Dronc), and two major effector caspases, Drosophila interleukin-1 converting

\footnotetext{
${ }^{1}$ Department of Biochemistry and Molecular Biology, University of Texas MD Anderson Cancer Center, 1515 Holcombe Boulevard, Unit 1000, Houston, TX, USA; ${ }^{2}$ University of Minnesota Interdisciplinary Informatics, OVPR, 1500 Gortner Avenue, Saint Paul, MN, USA and ${ }^{3}$ Howard Hughes Medical Institute, Rockefeller University, 1230 York Avenue, New York, NY, USA

${ }^{*}$ Corresponding author: A Bergmann, Department of Biochemistry and Molecular Biology, The University of Texas MD Anderson Cancer Center, 1515 Holcombe Boulevard, Unit 1000, Houston, TX 77030, USA. Tel: 1713834 6294; Fax: 1713834 6291; E-mail: abergman@mdanderson.org

Keywords: Drosophila; p53; Dp53; apoptosis

Abbreviations: ASK1, apoptosis signal-regulating kinase 1; Cas3 ${ }^{*}$, cleaved caspase-3; CDK, cyclin-dependent kinase; cep-1, c. elegans p53-like protein; dap dacapo; Dcp-1, death caspase-1; $\Delta \mathrm{N}$, Delta N; Df(3L)H99, deficiency (3L)H99; Dp53, Drosophila p53; DrlCE, Drosophila interleukin-1 converting enzyme; Dronc, Drosophila Nedd2-like caspase; ELAV, embryonic lethal abnormal vision; en, engrailed; ey, eyeless; FLP, flippase; FRT, flippase recombination target; GFP, green fluorescent protein; GMR, glass multimer reporter; GUS, GMR UAS; hid, head involution defective; hs, heat shock; JNK, Jun kinase; pros, prospero; ro, rough; rpr, reaper, SAM, sterile alpha motif; Svp, seven-up; TA, transactivating; tub, tubulin; TUNEL, terminal deoxynucleotidyl transferase dUTP nick end labeling; UAS, upstream activating sequence; XRE, X-ray response element; R1-R8, photoreceptor neurons 1-8

Received 31.8.09; revised 26.10.09; accepted 27.10.09; Edited by V De Laurenzi; published online 04.12.09
} 
enzyme (DrICE) and death caspase-1 (Dcp-1) (reviewed by $\mathrm{Xu}$ et $\mathrm{al}^{12}{ }^{12}$ ). In response to radiation-induced DNA damage, Dp53 activates the transcription of rpr to initiate apoptosis. ${ }^{6}$ In this process, hid is also induced, but the details are less clear. ${ }^{8,11,13}$

Expression of Dp53 in developing Drosophila eyes induces massive cell death. ${ }^{5,7}$ However, the Dp53-induced eye phenotype cannot be completely blocked by expression of p35, a potent inhibitor of DrICE and Dcp $-1,{ }^{5}$ suggesting that an effector caspase-independent mechanism of Dp53induced apoptosis may exist in Drosophila. There is precedence for a potential caspase-independent function of p53. Overexpression of the $C$. elegans p53 homolog (cep-1) caused wide-spread cell death independently of caspase activation. $^{14}$

In this study we further examined the phenotypes obtained by expression of Dp53 in Drosophila eyes. We show by mutant analysis that only hid, but not $r p r$, is required for Dp53induced apoptosis in this system. In addition, expression of Dp53 can activate the canonical caspase-dependent apoptosis pathway in Drosophila. Consistently, and in contrast to previous reports, we found that p35 can block cell death induced by expression of Dp53. However, inhibition of apoptosis does not rescue the Dp53-induced rough and small adult eye phenotype. We show that expression of Dp53 causes differentiation defects of various cell types, including photoreceptor neurons and cone cells, independently of its pro-apoptotic function. These differentiation defects imply that Dp53 may also have genetic features of mammalian p63 and p73 proteins. Intriguingly, expression of the p53 target gene, human p21, or its Drosophila homolog dap can suppress Dp53-induced cell death as well as cell differentiation defects. These findings reveal that Dp53 incorporates functions of multiple mammalian p53 family members and provide new insights into the pathways activated by Dp53.

\section{Results}

Expression of Dp53 induces cell death through the canonical apoptosis pathway in Drosophila eyes. Expression of Dp53 in the fly eye either directly under control of the eye-specific glass multimer reporter (GMR) promoter (GMR-Dp53) or using a modified upstream activating sequence (UAS)-Gal4 system (GMR-Gal4 GMR UAS (GUS)-Dp53; referred to as GMR $>$ GUS-Dp53) ${ }^{8}$ induces small and rough eyes with glossy appearance (Figures $1 \mathrm{a}-\mathrm{c})^{5,7,8}$ This eye ablation phenotype is induced specifically by $D p 53$ as it can be fully rescued by coexpression of a dominant-negative form of Dp53, Dp53 ${ }^{H 159 N}$ (see Ollmann et al. ${ }^{5}$ ), or by Dp53 RNAi (data not shown).

It has been reported that the eye ablation phenotype of GMR-Dp53 cannot be rescued by co-expression of the caspase inhibitor p35, an inhibitor of the effector caspases DrICE and Dcp $-1^{5}$ (see Figure $2 \mathrm{~h}$ ). This observation may suggest that GMR-Dp53 causes the eye ablation phenotype independently of caspase activation. Therefore, we examined the pro-apoptotic function of Dp53 in more detail. First, we labeled GMR-Dp53 and GMR $>$ GUS-Dp53 eye imaginal discs from late third instar larvae with an antibody detecting
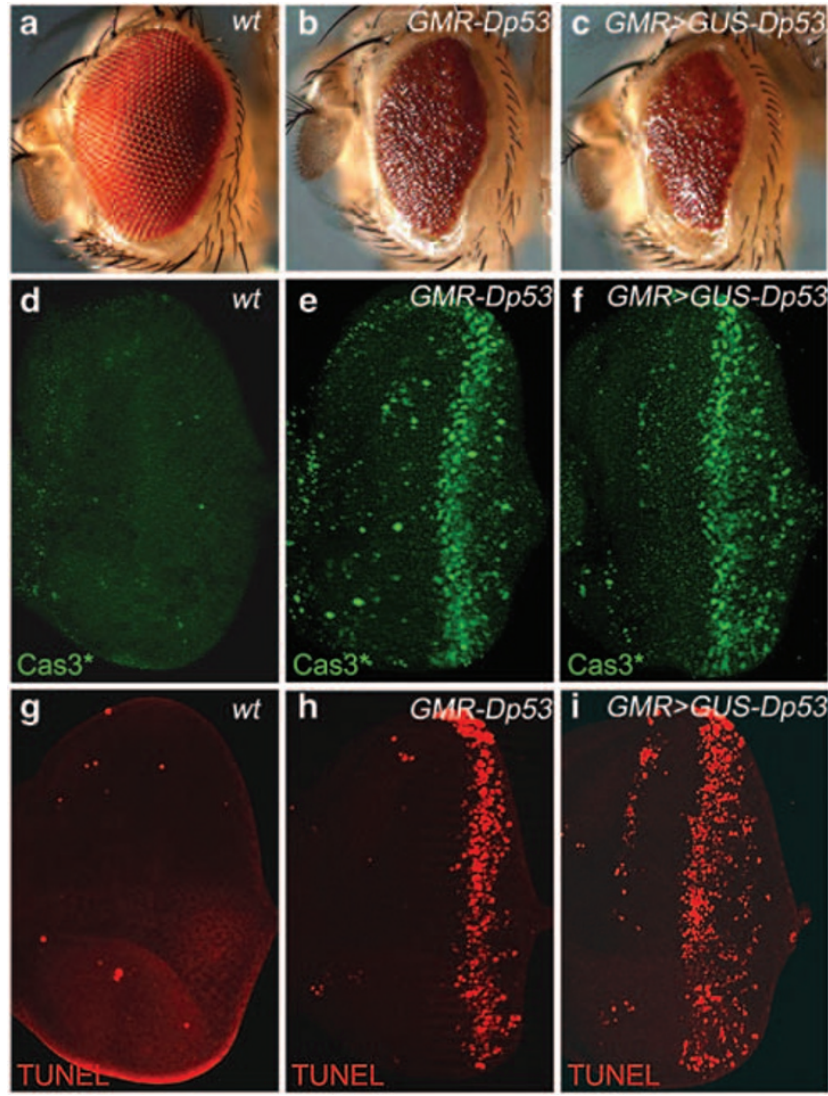

Figure 1 Expression of Dp53 induces massive cell death in Drosophila eyes. Shown are adult eyes $(\mathbf{a}-\mathbf{c})$ and late third instar larval eye imaginal discs $(\mathbf{d}-\mathbf{i})$. In this figure, and in the following figures, posterior is to the right. (a-c) Compared with wild type (wt) (a), expression of Dp53 under control of the GMR promoter (GMRDp53) (b) or the GMR-Gal4 driver (GMR > GUS-Dp53) (c) causes small adult eyes with rough and glossy appearance. (d) Wild-type eye disc labeled with anti-cleaved caspase-3 antibodies (Cas3*). A few cells are Cas3* positive. (e, f) GMR-Dp53 (e) and GMR > GUS-Dp53 (f) eye discs labeled with Cas3* antibodies. Massive cell death is induced in the posterior half of the eye disc in which GMR drives expression of Dp53. (g) Wild-type eye disc labeled with TUNEL. Only a few dying cells are labeled with TUNEL. (h, i) GMR-Dp53 (h) and GMR> GUS-Dp53 (i) eye discs labeled with the TUNEL assay. Massive dying cells are induced by expression of $D p 53$

activated caspases (cleaved caspase-3 (Cas3*)). The obtained labeling pattern (Figures $1 \mathrm{~d}-\mathrm{f}$ ) resembles the terminal deoxynucleotidyl transferase dUTP nick end labeling (TUNEL) pattern in these discs (Figures 1g-i) and corresponds to the expression domain of $G M R{ }^{15}$ Therefore, Dp53 can induce caspase activation.

Next, we asked whether the three major pro-apoptotic genes, rpr, hid and grim, are required for Dp53-induced cell death. Deficiency (3L)H99 (Df(3L)H99; referred to as H99), which deletes these three genes, ${ }^{16}$ was used for mosaic analysis. In late third instar GMR>GUS-Dp53 eye discs, Dp53-induced cell death, as detected by TUNEL, is completely blocked in $\mathrm{H} 99$ mutant clones (arrows, Figure 2a, $\mathrm{a}^{\prime}$ ). Furthermore, Dp53-induced cell death is also absent in mutant clones of the initiator caspase dronc (Figure $2 \mathrm{~b}, \mathrm{~b}^{\prime}$ ) or its adaptor ark (Figure 2c, $c^{\prime}$ ), which encode the apoptosome components of the canonical apoptotic pathway. ${ }^{17-21}$ 

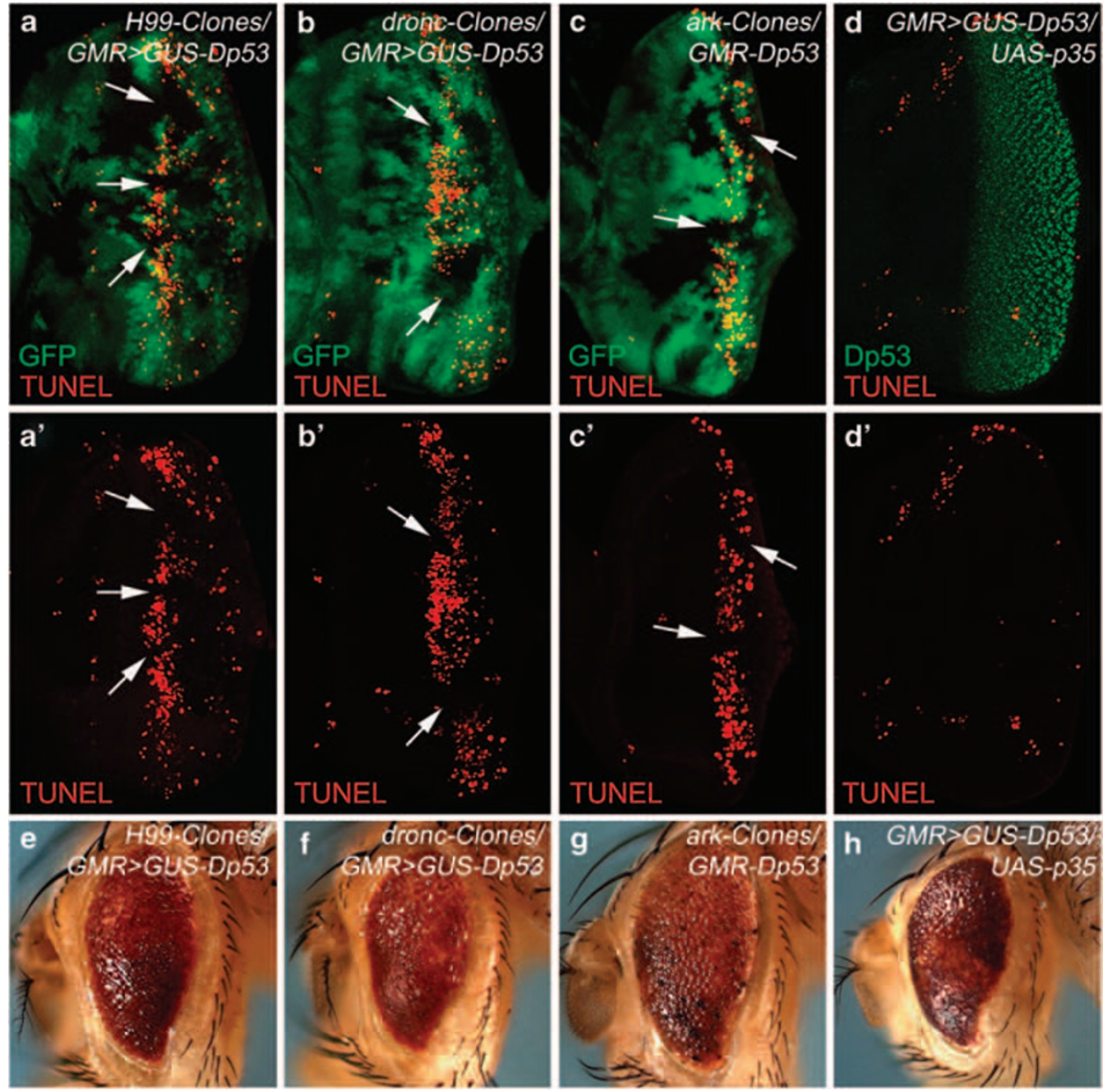

Figure 2 Dp53 induces cell death through the canonical apoptosis pathway in Drosophila. Shown are late third instar larval eye imaginal discs (a-d') and adult eyes (e-h) (a-c) Mosaic eye discs labeled with GFP (green) and TUNEL (red). Clones are marked by absence of GFP. H99 clones $\left(\mathbf{a}, \mathbf{a}^{\prime}\right)$ and dronc mutant clones (b, $\left.\mathbf{b}^{\prime}\right)$ are generated in GMR $>$ GUS-dp53 background and ark mutant clones are generated in GMR-Dp53 background (c, $\left.\mathbf{c}^{\prime}\right)$. Cell death induced by Dp53 is blocked in H99, dronc or ark mutant clones (arrows). (d, d') GMR > GUS-Dp53/UAS-p35 eye discs labeled with anti-Dp53 antibodies (green) and TUNEL (red). Dp53 is expressed in the posterior eye disc (d) and cell death induced by Dp53 is strongly suppressed by expression of P35 (d'). (e) H99 mutant mosaic eye in GMR $>$ GUS-Dp53 background. (f) dronc mutant mosaic eye in GMR > GUS-Dp53 background. (g) ark mutant mosaic eye in GMR-Dp53 background. (h) GMR > GUS-p53/UAS-p35 adult eye

These results indicate that the canonical Drosophila apoptotic pathway indeed mediates GMR-Dp53-induced cell death.

We next asked why expression of the caspase inhibitor p35 does not suppress the GMR-Dp53-induced eye phenotype ${ }^{5}$ (Figure 2h) and examined whether expression of p35 can block Dp53-induced apoptosis at the cellular level. Strikingly, simultaneous expression of $\mathrm{p} 35$ and Dp53 under control of the same GMR-GAL4 driver strongly inhibits Dp53-induced apoptosis in the developing eye disc (Figure $2 \mathrm{~d}, \mathrm{~d}^{\prime}$ ). The suppression of Dp53 by p35 is not restricted to eye imaginal discs and can also be observed in wing imaginal discs (Supplementary Figure S1).

Taken together, these data suggest that Dp53 activity triggers apoptosis through the canonical apoptotic pathway, including pro-apoptotic genes and activated caspases. Intriguingly, we also noticed that although GMR-Dp53induced cell death is completely blocked in H99, dronc or ark mutant clones, and is strongly suppressed by expression of p35, the resulting adult eyes are not or only partially rescued, as indicated by their rough and glossy appearance (compare Figures $2 \mathrm{e}-\mathrm{h}$ with Figures $1 \mathrm{~b}$ and $\mathrm{c}$ ). This is in striking contrast to the strong suppression of the GMR-hidand GMR-reaper-induced eye ablation phenotypes by loss of dronc and ark, or expression of p35. ${ }^{22-26}$ Therefore, this analysis raises two questions. First, which of the $\mathrm{H} 99$ genes, $r p r$, hid or grim, are required for caspase activation and apoptosis in GMR-Dp53 eye discs? Second, why is the eye ablation phenotype of GMR-Dp53 not rescued when apoptosis is blocked?

Hid is the major effector of Dp53-induced apoptosis in the Drosophila eye. To examine which pro-apoptotic genes mediate GMR-Dp53-induced apoptosis, we first examined the expression of hid in GMR-Dp53 eye discs. Compared with wild-type eye discs (Figure $\left.3 a-a^{\prime \prime \prime}\right)$, the protein level of Hid is strongly increased in the area in which GMR drives expression of Dp53 and apoptosis (Figure 3b, b" ; see also Figure 2d). Because Dp53 encodes a transcription factor, we 

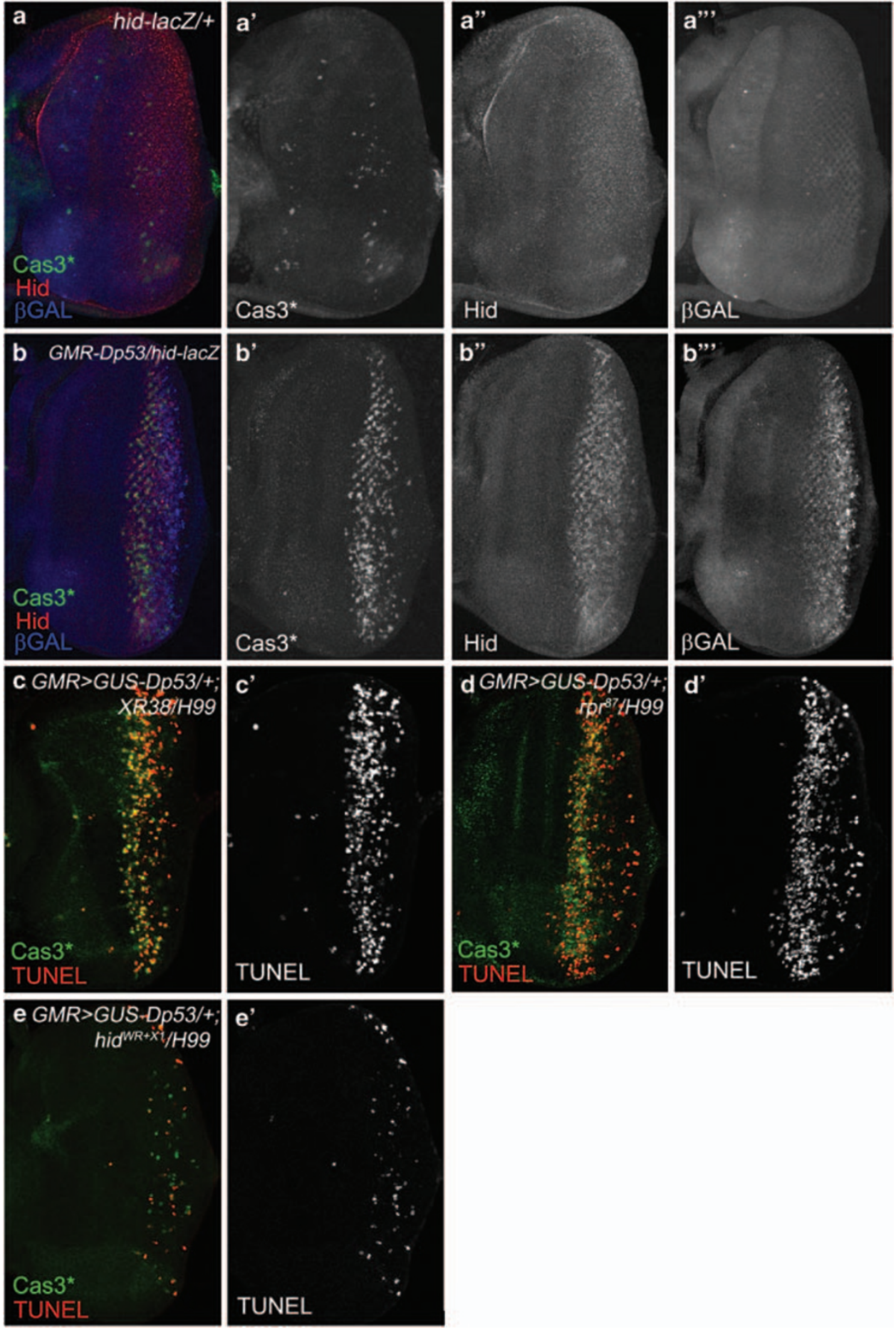

Figure 3 hid is the major effector of GMR-Dp53-induced apoptosis. Shown are late third instar larval eye imaginal discs labeled with Cas3* (green), anti-Hid antibodies (red) and $\beta$-GAL (blue) $(\mathbf{a}, \mathbf{b})$, or Cas3* (green) and TUNEL (red) (c, d). (a-a' $\left.\mathbf{a}^{\prime \prime \prime}\right)$ Wild-type disc containing the hid-lacZ reporter. Cell death and hid reporter expression are at

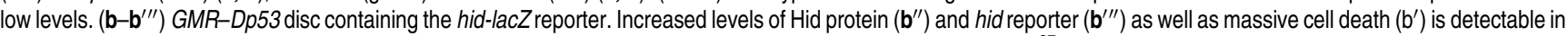
GMR-Dp53. (c, $\mathbf{c}^{\prime}$ and d, $\left.\mathbf{d}^{\prime}\right)$ GMR > GUS-Dp53 in rprhomozygous mutant background (XR38/H99 in $\mathbf{c}, \mathbf{c}^{\prime}$ and $r p r^{87} / H 99$ in d, $\mathbf{d}^{\prime}$ ). Dp53-induced cell death is not significantly altered in rpr mutants. (e, $\left.\mathbf{e}^{\prime}\right)$ GMR $>$ GUS-Dp53 in hid homozygous mutant background (hid $\left.{ }^{W R+X 1} / H 99\right)$. Dp53-induced cell death is strongly reduced as indicated by Cas3* and TUNEL labeling

tested whether this increase of Hid protein is due to increased hid transcription using a hid-lacZ reporter transgene (see Material and Methods). In GMR-Dp53 eye discs, hid is indeed transcriptionally induced (Figure 3b, $\mathrm{b}^{\prime \prime \prime}$ ). hid expression in response to Dp53 was not only present in developing eye discs but was also found in wing discs 
(Supplementary Figure S2b, $b^{\prime}$ ) when compared with controls (Supplementary Figure S2a).

Because rprhas been shown to be a direct target of Dp53 in response to X-ray-induced DNA damage, ${ }^{6}$ we also analyzed expression of rpr in GMR-Dp53. However, high background expression levels of the $r p r^{X R E}$-lacZ(X-ray response element (XRE)) reporter transgene prevented us from assessing $r p r$ expression in eye discs (data not shown). Nevertheless, we were able to detect increased reporter expression of the

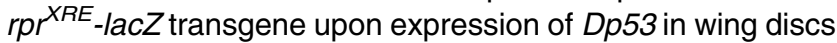
(Supplementary Figure S2d, $d^{\prime}$ ) compared with controls (Supplementary Figure S2c). Therefore, both hid and rpr are transcriptionally induced by Dp53 in imaginal eye and wing discs.

From the $\mathrm{H} 99$ mutant analysis (Figure 2a), we know that one or more of the $\mathrm{H} 99$ genes are important for GMR-Dp53induced apoptosis. To identify which gene is required for Dp53-induced apoptosis, we tested individual mutants. Surprisingly, loss of rpr by using a combination of deletions (H99/Df(3L)XR38), ${ }^{27}$ or a null mutant of $r p r, r p r^{87}$ (see Moon et $a l^{13}$ ), did not significantly affect the level of GMR-Dp53induced apoptosis (Figure $3 \mathrm{c}, \mathrm{c}^{\prime}$ and $\mathrm{d}, \mathrm{d}^{\prime}$ ). In contrast, null mutants of hid suppress most of GMR-Dp53-induced cell death (Figure $3 \mathrm{e}, \mathrm{e}^{\prime}$ ) indicating that $\mathrm{Hid}$ is the primary mediator of apoptosis induced by GMR-Dp53.

GMR-Dp53 causes cell differentiation defects independently of its apoptotic function. Although GMR-Dp53 induces apoptosis mainly through hid and its downstream canonical apoptotic pathway, the adult GMR-Dp53 eye phenotype cannot be rescued by blocking the apoptotic pathway (compare Figures $2 e-h$ with Figures $1 b$ and $c$ ). It is therefore reasonable to examine whether eye-specific expression of Dp53 can cause developmental defects other than apoptosis. It has been suggested that Dp53 expression may also cause differentiation defects. ${ }^{28}$ To analyze this possibility, we examined differentiation of various cell types in Dp53-expressing eye discs by using the cellular differentiation markers: Embryonic Lethal Abnormal Vision (ELAV; labels all photoreceptor neurons R1-R8), Rough (Ro; R2-R5) and Seven-up (Svp; R2, R5, R1 and R6). The expression of these differentiation markers in wild-type and GMR-Dp53 eye discs is shown in Supplementary Figure S3. Although differentiated photoreceptor neurons are slightly disorganized at the late larval stage, differentiation of all types of photoreceptor neurons, as visualized by these differentiation markers, appears largely normal in GMRDp53 (Supplementary Figure $S 3 b-b^{\prime}$ ). In contrast, the numbers of $\mathrm{R} 7$ photoreceptor neurons and cones cells labeled by the markers Prospero (Pros) and Cut, respectively, are strongly reduced compared with wild type (Figures $\left.4 a-d^{\prime}\right)$. Therefore, expression of Dp53 under control of the GMR promoter affects differentiation of $\mathrm{R} 7$ and cone cells. To further examine the differentiation defect, GMR-Dp53 pupal eye discs were analyzed. In pupal GMR-Dp53 eye discs, ommatidia are severely misorganized, as indicated by enlarged interommatidial space, ommatidial fusions (arrows, Figure 4f) and reduced number of cone cells in each ommatidium. Altogether, these data indicate that
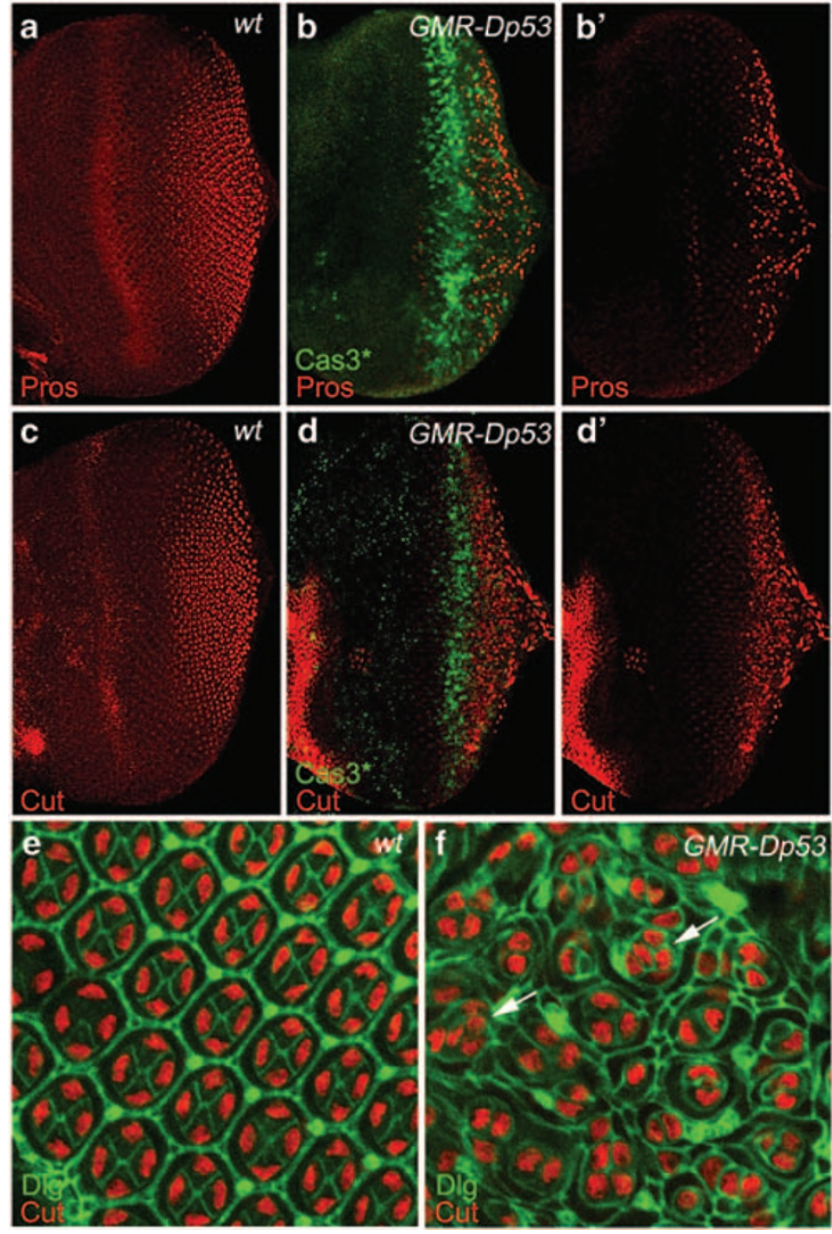

Figure 4 Differentiation of R7 and cone cells is disrupted in GMR-Dp53 eye discs. Shown are late third instar eye imaginal discs $\left(\mathbf{a}-\mathbf{d}^{\prime}\right)$ and mid-pupal eye discs $(\mathbf{e}, \mathbf{f}) .\left(\mathbf{a}-\mathbf{b}^{\prime}\right)$ Eye discs labeled with the R7 photoreceptor marker Pros (red) and Cas3 $^{*}$ (green). Compared with wild type (a), the number of R7 cells as indicated by Pros labeling is strongly reduced in GMR-Dp53 discs (b, $\left.\mathbf{b}^{\prime}\right)$. (c- $\left.\mathbf{d}^{\prime}\right)$ Eye discs labeled with the cone cell marker Cut (red) and Cas3* (green). Compared with wild type (c), the number of cone cells as indicated by Cut staining is strongly reduced in GMR-Dp53 discs (d, $\mathbf{d}^{\prime}$ ). (e, f) Pupal discs labeled with Cut (red) and the cellular membrane marker Dlg (green). In wild type (e), ommatidia are well organized and contain four cone cells each. In contrast, in GMR-Dp53 discs (f), the global organization of ommatidia is severely disrupted. Loss of cone cells in some ommatidia and ommatidial fusion (arrows), as indicated by aggregated cone cells, are observed

GMR-Dp53 causes differentiation defects in developing Drosophila eyes.

Because Dp53 induces apoptosis, we wondered whether these differentiation defects are caused by the pro-apoptotic function of Dp53, and analyzed cell differentiation in Dp53expressing, but apoptosis-deficient, background. In control experiments, inhibition of apoptosis in otherwise wild-type background does not affect cell differentiation (data not shown). Although cell death is blocked in H99 or dronc mutant tissues (see Figures $2 a$, and $b$ and $5 b$ ), R7 photoreceptor differentiation is not rescued in GMR-Dp53 eye discs (Figure $5 \mathrm{a}, \mathrm{b}^{\prime}$ ). Similar results were obtained during pupal development (Figures $5 \mathrm{c}-\mathrm{e}^{\prime}$ ). This analysis suggests that 

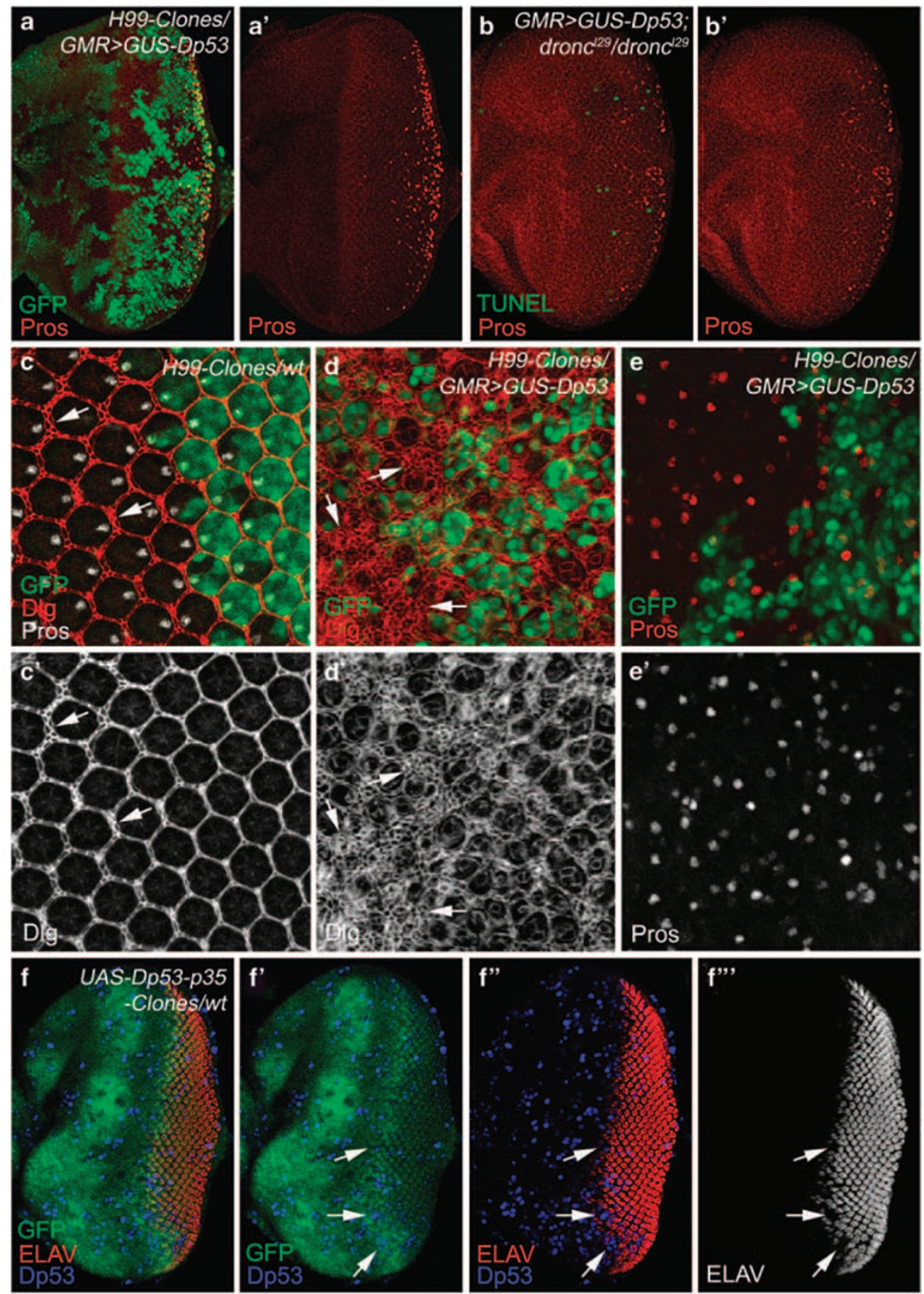

Figure 5 GMR-Dp53 induces cell differentiation defects independently of its pro-apoptotic role. Shown are late third instar eye imaginal discs (a-b $\mathbf{b}^{\prime}$ and $\left.\mathbf{f}-\mathbf{f}^{\prime \prime \prime}\right)$ and $\mathrm{mosaic}$ mid-pupal eye discs $\left(\mathbf{c}-\mathbf{e}^{\prime}\right)$. (a, $\left.\mathbf{a}^{\prime}\right)$ A GMR > GUS-Dp53 disc with H99 mutant clones labeled with GFP (green) and Pros (red). H99 clones are marked by absence of GFP. The number of R7 cells does not increase in H99 clones in which apoptosis is blocked. (b, $\left.\mathbf{b}^{\prime}\right)$ A homozygous dronc mutant GMR $>$ GUS-Dp53 disc labeled with TUNEL (green) and Pros (red). Dp53-induced cell death is strongly suppressed with only a few dying cells left (b). However, despite inhibition of apoptosis, R7 differentiation is not restored $\left(\mathbf{b}\right.$ and $\left.\mathbf{b}^{\prime}\right)$. (c, $\mathbf{c}^{\prime}$ ) Mosaic wild-type pupal disc labeled with GFP (green), Dlg (red) and Pros (gray). H99 clones are marked by absence of GFP. Although the number of interommatidial cells is increased in $\mathrm{H} 99$ clones (arrows), the ommatidial organization is normal and there is a single R7 cell (gray) in each ommatidium (c). (d- $\mathbf{e}^{\prime}$ ) GMR > GUS-Dp53 pupal discs with H99 mutant clones labeled with GFP (green) and Dlg (red) (d), or GFP (green) and Pros (red) (e). H99 clones are marked by absence of GFP. A strongly increased number of interommatidial cells (arrows) is observed in $\mathrm{H} 99$ clones (d, $\mathbf{d}^{\prime}$; arrows). Importantly, the disrupted organization of ommatidia (d'), and the disorganization and reduced number of R7 cells ( $\left.\mathbf{e}^{\prime}\right)$ in GMR > GUS-Dp53 discs is not rescued in H99 clones. (f- $\mathbf{f}^{\prime \prime \prime}$ ) Mosaic discs labeled with GFP (green), the neuronal marker ELAV (red) and anti-Dp53 antibodies (blue). Clones simultaneously expressing Dp53 and p35 are marked by absence of GFP and by anti-Dp53 labeling (blue). In these clones, although cell death is blocked by p35, differentiation of photoreceptor neurons is blocked as indicated by lack of ELAV staining (arrows) 
expression of Dp53 causes differentiation defects independently of its pro-apoptotic role.

Next, we analyzed why differentiation of $\mathrm{R} 7$ and cone cells was affected by $G M R-D p 53$, but not that of other photoreceptor neurons. $\mathrm{R} 7$ and cone cells are the last cell types to be specified in the larval eye disc. In contrast, photoreceptors $\mathrm{R} 8, \mathrm{R} 2, \mathrm{R} 3, \mathrm{R} 4$ and R5 are specified earlier at around the time when GMR induces expression of Dp53. Thus, it is possible that Dp53 is expressed too late to block differentiation of photoreceptor neurons R8 and R2-R5. We examined this possibility by inducing Dp53-expressing clones at earlier stages. To block Dp53-induced apoptosis and thus to obtain Dp53-expressing clones, the caspase inhibitor p35 was expressed simultaneously with Dp53. Under these conditions, all types of photoreceptor neurons as indicated by ELAV labeling are missing in Dp53/p35-expressing tissues (arrows,Figure $\left.5 f-f^{\prime \prime \prime}\right)$. Expression of p35 alone does not affect photoreceptor and cone cell differentiation (data not shown). Thus, these observations suggest that Dp53 can only block differentiation if it is expressed before the onset of differentiation. In summary, these data show that expression of Dp53 can interfere with differentiation of all cell types in developing Drosophila eyes independently of its pro-apoptotic function.

\section{Human p21 and Drosophila Dap suppress both GMR- Dp53-induced apoptosis and cell differentiation} defects. It has been reported that expression of human p21 can suppress Dp53-induced apoptosis in Drosophila. ${ }^{5}$ Because we showed above that Dp53-induced cell differentiation defects and apoptosis are independent of each other (see above), we further examined whether and how human p21 can rescue GMR-Dp53-induced phenotypes in more detail. Expression of p21 under GMR control (GMR-p21) causes a rough eye phenotype due to decreased cell proliferation (Figure 6a). ${ }^{29}$ GMR-p21 does not or only mildly induce cell death and does not affect photoreceptor differentiation (Figures $6 \mathrm{~b}$ and $\mathrm{c}$ ). Consistently

Figure 6 Expression of human p21 or Drosophila Dap suppresses both GMRDp53-induced apoptosis and cell differentiation defects. (a) GMR-p21/+ adult eye. A little roughening is visible. (b, c) Control larval eye discs of GMR-p21/+ at the late third instar stage labeled with $\operatorname{Cas}^{*}(\mathbf{b})$ and the neuronal marker ELAV (c). GMR-p21 induces no or little apoptosis (b, arrow), and differentiation of photoreceptor neurons is largely normal (c). (d) Adult eye of GMR-Dp53/GMRp21. Expression of human p21 rescues Dp53-induced eye phenotype (compared with Figure 1b). (e, f) Late third instar eye discs of GMR-Dp53/GMR-p21 labeled with Cas3 $^{*}$ (e) or Pros (f). Dp53-induced cell death is largely suppressed (e, arrowhead) and the number of R7 cells is restored (f). (g) Adult eye of GMR > GUS-Dp53/UAS-dap. Expression of dacapo (dap) partially rescues the Dp53-induced eye ablation phenotype (compared with Figure 1c). (h, i) Late third instar eye discs of GMR > GUS-Dp53/UAS-dap labeled with Cas3* (h) or Pros (i). Dp53-induced cell death is partially suppressed ( $h$, arrowhead) and the number of R7 cells is partially restored (i). (j) Late third instar eye disc of GMR-Dp53/GMRp21 labeled with anti-Hid antibody. The level of Dp53-induced Hid is reduced in response to expression of human p21 (compared with Figure $\left.3 b^{\prime \prime}\right)$. (k) Expression of Dp53 suppresses cell differentiation independently of its roles in apoptosis. Dp53 activates apoptosis mainly through the pro-apoptotic gene Hid and its downstream canonical apoptosis pathway in Drosophila. Expression of human p21 or its Drosophila homolog Dacapo (Dap) suppresses both p53-induced cell differentiation defects and Hid-induced cell death with previous observations, GMR-p21 suppresses GMRDp53-induced apoptosis in larval eye discs (Figure 6e). More importantly, in contrast to $p 35, G M R-p 21$ rescues both the R7 differentiation defect in larval eye discs and the eye ablation phenotype of GMR-Dp53 adults (Figures $6 \mathrm{~d}$ and f). Similarly, although to a lesser extent, expression of the Drosophila homolog of p21, dacapo (dap), ${ }^{30,31}$ also suppresses both Dp53-induced cell death and cell differentiation defects (Figures 6g-i). As described above, because expression of Dp53 induces expression of the apoptotic gene hid, we examined whether GMR-p21 can modulate GMR-Dp53-induced expression of hid. Indeed, the protein level of Hid is reduced when human p21 is co-expressed in Dp53-expressing eyes (compare Figure 6j with Figure $3 \mathrm{~b}^{\prime \prime \prime}$ ).

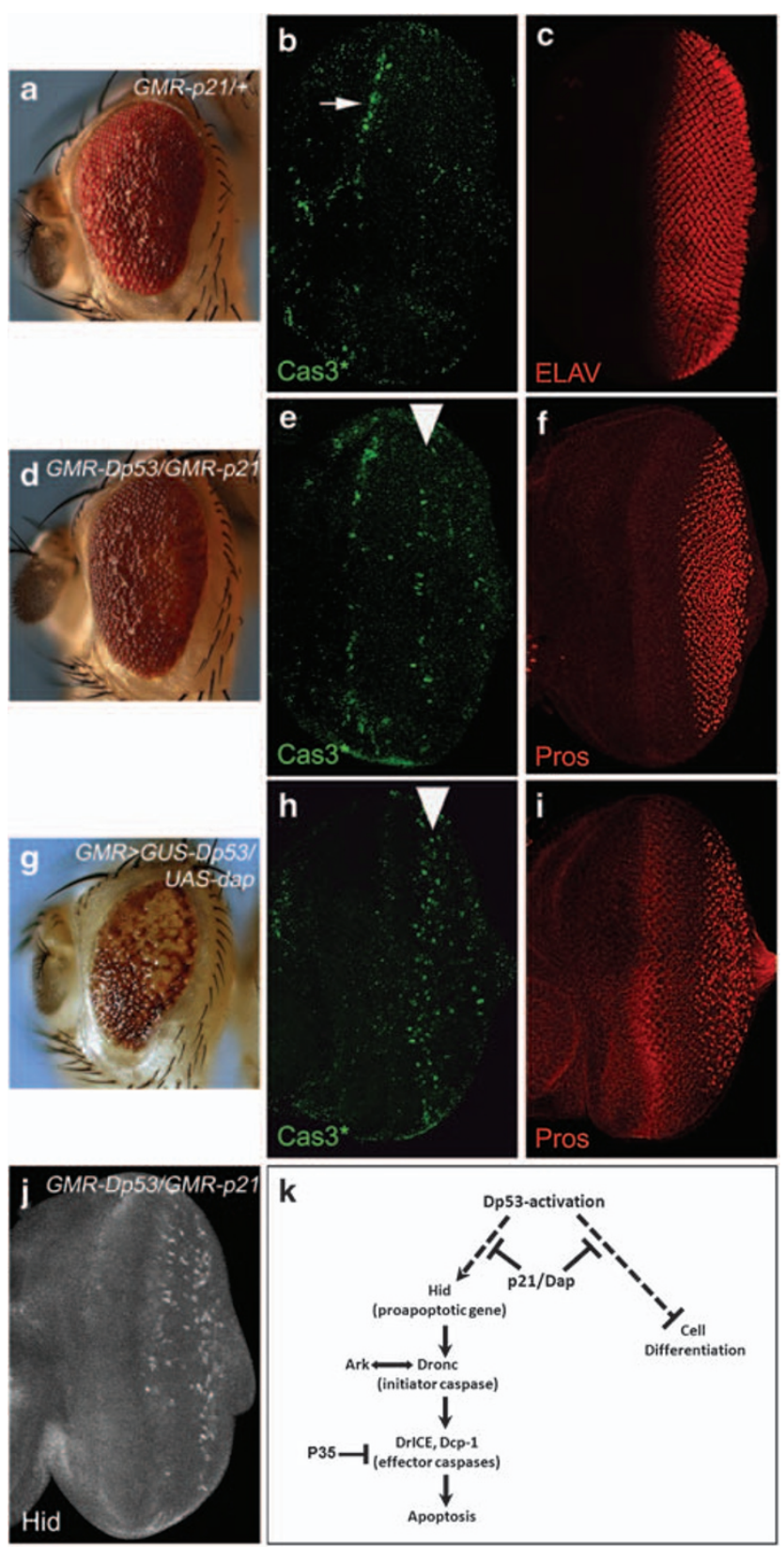


This observation suggests that p21 interferes with Dp53 upstream of hid to suppress Dp53-induced apoptosis in Drosophila (Figure 6k).

\section{Discussion}

In this study, we used the developing Drosophila eye as an in vivo model to analyze the function of Dp53 at the cellular level. Our study makes three important points. First, Hid is the major effector of GMR-Dp53-induced apoptosis, triggering the canonical caspase-dependent apoptotic pathway. Second, Dp53 induces differentiation defects of all cell types in the eye. This activity is independent of the pro-apoptotic role of Dp53, and reminiscent of mammalian p63 and p73. Third, these dual roles of Dp53 can be inhibited by expression of human p21 or its Drosophila homolog dap. In the following, we discuss these observations in detail.

Hid is the major effector of GMR-Dp53-induced apoptosis. Previous studies of radiation-induced cell death have shown that the pro-apoptotic genes rpr and hid are transcriptionally induced by Dp53.6,8,11,32,33 The fast induction (within $30 \mathrm{~min}$ ) of hid and rpr suggested that they may be direct targets of Dp53. ${ }^{8}$ A radiation-responsive enhancer containing a typical p53-binding consensus site was identified in the upstream regulatory region of $\mathrm{rpr}^{6}{ }^{6}$ GMR-Dp53 induces reporter expression from the same radiation-responsive enhancer, suggesting that induction of rpr by Dp53 is independent of context. However, despite expression of $r p r$, loss of $r p r$ does not significantly influence GMR-Dp53-induced apoptosis. In contrast, complete loss of hid significantly abrogated GMR-Dp53-induced apoptosis, suggesting that Hid is the major mediator of GMR-Dp53. This is consistent with previous findings that heterozygosity of hid partially suppresses radiation-induced apoptosis. ${ }^{8}$ Therefore, it seems that Dp53 - whether its expression is induced by irradiation or by the heterologous GMR promoter - induces apoptosis by similar molecular mechanisms.

It is unclear why hid has a more important role in GMRDp53-induced apoptosis than $r p r$ in this system. Simple expression of rpr may not be sufficient for apoptosis induction and additional activation may be required. However, this possibility seems unlikely because expression of rpr from the GMR promoter is sufficient to induce apoptosis. ${ }^{34}$ Alternatively, the developing eye may be more prone to hid-induced apoptosis because those cells that die by developmental apoptosis in the eye die primarily by hid-induced apoptosis. ${ }^{35}$ Such a tissue-specific requirement has also been reported for $r p r$, which is required for apoptosis of abdominal neuroblasts in the central nervous system. ${ }^{27}$ Thus, it will be interesting to analyze the pro-apoptotic requirements of Dp53 in other tissues including neuroblasts.

Dp53 has a conserved function in regulating cell differentiation. Our analysis indicates that Dp53 blocks cell differentiation of photoreceptor neurons and cone cells independently of its pro-apoptotic role. Interestingly, Dp53 can only block differentiation if it is expressed before the onset of differentiation. Notably, p53, p63 and p73 have also been implicated in the control of cell differentiation (reviewed in Vousden and Prives ${ }^{1}$ and Stiewe ${ }^{3}$ ). However, in the cases reported, the expression of the p53 family members in undifferentiated cells actually induces differentiation instead of inhibiting it, as shown in this study for Dp53. For example, the TA isoform of mouse p53 induces differentiation of mouse embryonic stem cells. ${ }^{3,4}$ Nevertheless, the antagonizing $\Delta \mathrm{N}$ isoforms of $\mathrm{p} 63$ and $\mathrm{p} 73$ have been found to promote stem cell proliferation. For example, $\Delta$ Np63 is highly expressed in epidermal stem cells, and loss of p63 triggers these cells to terminally differentiate, suggesting that $\Delta$ Np63 inhibits differentiation (reviewed in Stiewe ${ }^{3}$ and Murray-Zmijewski et al. ${ }^{4}$ ). Similarly, expression of $\Delta \mathrm{Np} 73$ inhibits myogenic differentiation. ${ }^{3,4} \Delta \mathrm{Np} 63$ and $\Delta \mathrm{Np} 73$ can also interfere with p53-induced differentiation programs. ${ }^{3,4}$

Importantly, the Dp53 gene also has an internal promoter ${ }^{36}$ and the originally identified Dp53gene, including the one used in this study, actually corresponds to the $\Delta \mathrm{N}$ isoform of Dp53. ${ }^{36}$ Therefore, our finding that expression of Dp53 suppresses cell differentiation is consistent with the inhibitory role of $\Delta \mathrm{N}$ isoforms in cell differentiation. Therefore, Dp53 has similar genetic properties to mammalian $\Delta \mathrm{Np} 63$ and $\Delta \mathrm{Np} 73$ isoforms. This statement is also supported by the observation that mammalian cells lacking p63 and p73 are unable to induce DNA damage-induced cell-cycle arrest, ${ }^{10}$ similar to Dp53. However, both mammalian p53 and Drosophila Dp53 lack the sterile alpha motif (SAM) domain at the C-terminus, which is characteristic for p63 and p73. ${ }^{37}$ The SAM domain supports oligomerization of p63 and p73. The absence of the SAM domain may indicate that Dp53 is more related with mammalian p53 rather than with p63 and p73. Nevertheless, BLAST searches with Dp53 revealed higher similarity with mammalian p63 and p73 than with p53. ${ }^{37}$ Furthermore, it was recently shown that the SAM domain of Dp53 was replaced during evolution by a helix domain, which also supports oligomerization. $^{38}$ Thus, both genetically and functionally, Dp53 resembles p63 and p73 more than p53.

p21 and Dacapo antagonize an early step of Dp53 activation. Depending on the cell type, expression of human p21 can suppress p53-induced apoptosis (reviewed by Janicke et al. ${ }^{39}$ ). Because after DNA damage $\mathrm{p} 53$ induces cell-cycle arrest through induction of p21, it is thought that p21-mediated suppression of p53-induced apoptosis would give cells the opportunity to repair damaged DNA first, before induction of apoptosis, depending on the extent of DNA damage. ${ }^{39}$ We extend these observations further and show in this study that human p21 not only suppresses Dp53induced apoptosis, but also suppresses the Dp53-induced block of cell differentiation. Remarkably, the suppression of Dp53-induced phenotypes is more efficient by human p21 than by Drosophila Dap. It is surprising that this control of Dp53 activity is conserved in flies, given that Dp53 is not required for radiation-induced cell-cycle arrest and also does not induce dap expression..$^{5,6,8,11}$ However, the fact that Dap exerts at least some anti-Dp53 activity suggests that human p21 did not acquire this activity recently in evolution. It rather seems that Dap may have partially lost it because it is not induced by $\mathrm{Dp53}$ and thus there was no selective pressure for Dap to maintain its anti-Dp53 activity during evolution, and hence the weaker suppression. 
The molecular mechanisms by which $\mathrm{p} 21$ suppresses $\mathrm{p} 53-$ induced apoptosis are unclear and somewhat contradictory (reviewed in Janicke et al. $^{39}$ ). According to several studies, the anti-apoptotic function of p21 seems to be mediated through binding and inhibition of caspase-3, apoptosis signalregulating kinase 1 (ASK1), Jun kinase (JNK), p38 and CDKs. ${ }^{39}$ However, our study provides three lines of evidence that p21-mediated suppression of Dp53 occurs upstream of hid expression. First, the Dp53-induced block of cell differentiation, which is independent of hid, is suppressed by p21. Second, Dp53-induced expression of hid is strongly reduced by $\mathrm{p} 21$. Finally, the apoptotic phenotype of GMR-hid is not affected by co-expression of p21 (data not shown). Thus, these data support the notion that p21 and Dap suppress GMR-Dp53 upstream of hid. p21 does not affect the protein levels of Dp53 in GMR-Dp53 (data not shown), suggesting that p21 does not interfere with Dp53 expression, translation or stability.

Because p21 suppresses Dp53 at a very early step and because Dp53 is thought to directly bind to the hid promoter, ${ }^{8}$ we would suggest that p21 directly interferes with the ability of Dp53 to induce gene expression. Alternatively, it is also possible that the suppression of Dp53 by p21 is indirect, and that Dp53 requires a cell-cycle-competent environment for hid expression. Further studies are needed to clarify these questions.

In summary, these findings reveal that Dp53 incorporates functions of multiple mammalian p53 family members and provide new insights into the pathways activated by Dp53. It will now be interesting to identify the mechanisms by which Dp53 inhibits cell differentiation and how p21 overcomes it.

\section{Materials and Methods}

Fly strains and crosses. All stocks were reared at room temperature. The GMR promoter is described in Ellis et al. ${ }^{15}$ dronc $^{129},{ }_{22}$ ark $^{G 8},{ }^{24} \mathrm{Df}(3 L) H 99,{ }^{16}$ $\operatorname{Df}(3 L) X R 38^{27}$ hid $^{W R+X 1},{ }^{26} \mathrm{GMR}-\mathrm{p} 21^{29}$ and UAS-dap ${ }^{30}$ are as described previously. The engailed-GAL4 (en-GAL4), eyeless-flippase (ey-FLP), heat shock-flippase (hs-FLP), flippase recombination target (FRT)80 P[ubi-GFP], UAS-p35, GMR-Dp53, GMR > GUS-Dp53 and UAS-Dp53 ${ }^{H 159 N}$ were obtained from the Bloomington Stock Center (Indiana University, Bloomington, IN, USA). The GMR-p21 and UAS-dap lines were kindly provided by I Hariharan.

\section{Generation of the hid-lacZ and $r p r^{X R E}{ }_{-l a c Z}$ reporter lines}

hid-lacZ. The $30 \mathrm{~kb}$ genomic DNA upstream of the hid start site was cloned in three parts with approximately $10 \mathrm{~kb}$ each and a small region of overlap around 100-200 bp. These three DNA fragments were then cloned and inserted into a Drosophila reporter plasmid, pCaSpeR-hs43-lacZ, respectively. Each reporter construct was named based on the distance upstream of the hid start site, for example, hid ${ }^{20-10}-$ lac $Z$ contains regulatory DNA $10-20 \mathrm{~kb}$ upstream of the start site. Transgenic flies were then generated using standard procedures. The $h_{i{ }^{20-10}}$-lacZ reporter was found to respond in GMR-Dp53 eye discs (Figure 3b) and was used in this study as the hid reporter.

$r p r^{X R E}-$ lacZ. Previous work led to the identification of a 2.2-kb Ndel-Bgll genomic interval necessary for the activation of $r p r$ gene expression in response to X-ray and UV radiation exposure (Lamblin and Steller, unpublished). Using sequence-specific primers with BamHI restriction sites, the Ndel-Bgll $2.2 \mathrm{~kb}$ X-ray interval was amplified by PCR and cloned directly into the BamHI site of $1.3 \mathrm{rpr}$-LacZ reporter vector ${ }^{40}$ for $P$ element-mediated germline transformation. Transgenic embryos submitted to X-ray treatment showed a strong upregulation of lacZ expression and activity in response to X-ray and UV radiation exposure (Lamblin and Steller, unpublished).

Mosaic analysis. To examine H99, dronc or ark clones in Dp53-expressing eye discs, late third instar larvae of the following genotype were analyzed:
(1) w eyFLP; GMR> GUS-Dp53/+; H99 FRT80/P[ubi-GFP] FRT80; (2) $w$; GMR> GUS-Dp53/+; dronc ${ }^{29}$ FRT80/P[ubi-GFP] FRT80; and (3) w; ark ${ }^{G 8}$ FRT42/P[ubi-GFP] FRT42; GMR-Dp53/+. For mosaic analysis with clones expressing Dp53 and p35 simultaneously, larvae of the following genotype were heat shocked for $30 \mathrm{~min}$ at $37^{\circ} \mathrm{C}$, raised at room temperature and analyzed $48 \mathrm{~h}$ later: $w$ hsFLP/+; tubulin (tub) > GFP > GAL4/UAS-p35; UAS-Dp53/+. In each of these experiments, more than 20 representative clones were analyzed.

Immunohistochemistry. Eye-antennal imaginal discs from late third instar larvae or mid-pupa (45-50 h after puparium formation) were dissected and labeled with the following antibodies: rabbit anti-Dlg and mouse anti-Rough (kindly provided by $\mathrm{K}$ Choi), rabbit anti-Seven-up (a gift from $\mathrm{R}$ Schulz), rabbit anti-cleaved caspase3 (Cell Signaling Technology, Danvas, MA, USA), mouse anti-Dp53, rat anti-ELAV, mouse anti-Pros, mouse anti-Cut and mouse anti- $\beta$ GAL (all obtained from the DSHB, University of lowa, lowa City, IA, USA). Secondary antibodies were donkey Fab fragments from Jackson ImmunoResearch (West Grove, PA, USA). The TUNEL assay kit is from Roche (Indianapolis, IN, USA). Images were taken with either a Zeiss Axiolmager equipped with ApoTome technology (Carl Zeiss Microlmaging, Thornwood, NY, USA) or a confocal microscope.

\section{Conflict of interest}

The authors declare no conflict of interest.

Acknowledgements. We apologize to our colleagues whose work could not be cited because of space restrictions. We thank John Abrams, Michael Brodsky, Kwang Choi, Bruce Hay, Iswar Hariharan, Hyung Don Ryoo, Robert Schulz, Kristin White, the Bloomington Stock Center in Indiana and the Developmental Studies Hybridoma Bank (DSHB) in lowa for fly stocks and reagents. This work was supported by the NIH (R01GM068018, R01GM081543 and R01GM074977 to AB, and R01GM60124 to HS). The content is solely the responsibility of the authors and does not represent the official views of the $\mathrm{NIH}$. We gratefully acknowledge support by the Robert A Welch Foundation (G-1496).

1. Vousden $\mathrm{KH}$, Prives C. Blinded by the light: the growing complexity of p53. Cell 2009; 137: 413-431.

2. Pietsch EC, Sykes SM, McMahon SB, Murphy ME. The p53 family and programmed cell death. Oncogene 2008; 27: 6507-6521.

3. Stiewe T. The p53 family in differentiation and tumorigenesis. Nat Rev 2007; 7: 165-168.

4. Murray-Zmijewski F, Lane DP, Bourdon JC. p53/p63/p73 isoforms: an orchestra of isoforms to harmonise cell differentiation and response to stress. Cell Death Differ 2006; 13: 962-972.

5. Ollmann M, Young LM, Di Como CJ, Karim F, Belvin M, Robertson S et al. Drosophila p53 is a structural and functional homolog of the tumor suppressor p53. Cell 2000; 101: 91-101.

6. Brodsky MH, Nordstrom W, Tsang G, Kwan E, Rubin GM, Abrams JM. Drosophila p53 binds a damage response element at the reaper locus. Cell 2000; 101: 103-113.

7. Jin S, Martinek S, Joo WS, Wortman JR, Mirkovic N, Sali A et al. Identification and characterization of a p53 homologue in Drosophila melanogaster. Proc Natl Acad Sc USA 2000; 97: 7301-7306.

8. Brodsky MH, Weinert BT, Tsang G, Rong YS, McGinnis NM, Golic KG et al. Drosophila melanogaster MNK/Chk2 and p53 regulate multiple DNA repair and apoptotic pathways following DNA damage. Mol Cell Biol 2004; 24: 1219-1231.

9. Yamada $Y$, Davis KD, Coffman CR. Programmed cell death of primordial germ cells in Drosophila is regulated by p53 and the Outsiders monocarboxylate transporter. Development 2008; 135: 207-216.

10. Flores ER, Tsai KY, Crowley D, Sengupta S, Yang A, McKeon F et al. p63 and p73 are required for p53-dependent apoptosis in response to DNA damage. Nature 2002; 416: 560-564.

11. Akdemir F, Christich A, Sogame N, Chapo J, Abrams JM. p53 directs focused genomic responses in Drosophila. Oncogene 2007; 26: 5184-5193.

12. Xu D, Woodfield SE, Lee TV, Fan Y, Antonio C, Bergmann A. Genetic control of programmed cell death (apoptosis) in Drosophila. Fly 2009; 3: 78-90.

13. Moon NS, Di Stefano L, Morris EJ, Patel R, White K, Dyson NJ. E2F and p53 induce apoptosis independently during Drosophila development but intersect in the context of DNA damage. PLOS Genet 2008; 4: e1000153.

14. Derry WB, Putzke AP, Rothman JH. Caenorhabditis elegans p53: role in apoptosis, meiosis, and stress resistance. Science (New York, NY) 2001; 294: 591-595.

15. Ellis MC, O'Neill EM, Rubin GM. Expression of Drosophila glass protein and evidence for negative regulation of its activity in non-neuronal cells by another DNA-binding protein. Development 1993; 119: 855-865. 
16. White K, Grether ME, Abrams JM, Young L, Farrell K, Steller H. Genetic control of programmed cell death in Drosophila. Science (New York, NY) 1994; 264: 677-683.

17. Dorstyn L, Colussi PA, Quinn LM, Richardson H, Kumar S. DRONC, an ecdysone-inducible Drosophila caspase. Proc Natl Acad Sci USA 1999; 96: 4307-4312.

18. Zhou L, Song Z, Tittel J, Steller H. HAC-1, a Drosophila homolog of APAF-1 and CED-4 functions in developmental and radiation-induced apoptosis. Mol Cell 1999; 4: 745-755.

19. Kanuka H, Sawamoto K, Inohara N, Matsuno K, Okano H, Miura M. Control of the cell death pathway by Dapaf-1, a Drosophila Apaf-1/CED-4-related caspase activator. Mol Cell 1999; 4: 757-769

20. Rodriguez A, Oliver $\mathrm{H}$, Zou $\mathrm{H}$, Chen $\mathrm{P}$, Wang $\mathrm{X}$, Abrams JM. Dark is a Drosophila homologue of Apaf-1/CED-4 and functions in an evolutionarily conserved death pathway. Nat Cell Biol 1999; 1: 272-279.

21. Mills K, Daish T, Harvey KF, Pfleger CM, Hariharan IK, Kumar S. The Drosophila melanogaster Apaf-1 homologue ARK is required for most, but not all, programmed cell death. J Cell Biol 2006; 172: 809-815.

22. Xu D, Li Y, Arcaro M, Lackey M, Bergmann A. The CARD-carrying caspase Dronc is essential for most, but not all, developmental cell death in Drosophila. Development 2005; 132: 2125-2134.

23. Daish TJ, Mills K, Kumar S. Drosophila caspase DRONC is required for specific developmental cell death pathways and stress-induced apoptosis. Dev Cell 2004; 7: 909-915.

24. Srivastava M, Scherr H, Lackey M, Xu D, Chen Z, Lu J et al. ARK, the Apaf-1 related killer in Drosophila, requires diverse domains for its apoptotic activity. Cell Death Differ 2007; 14: 92-102.

25. Xu D, Wang Y, Willecke R, Chen Z, Ding T, Bergmann A. The effector caspases drICE and dcp-1 have partially overlapping functions in the apoptotic pathway in Drosophila. Cell Death Differ 2006; 13: 1697-1706.

26. Grether ME, Abrams JM, Agapite J, White K, Steller H. The head involution defective gene of Drosophila melanogaster functions in programmed cell death. Genes Dev 1995; 9: 1694-1708.

27. Peterson C, Carney GE, Taylor BJ, White K. reaper is required for neuroblast apoptosis during Drosophila development. Development 2002; 129: 1467-1476.
28. Kondo S, Senoo-Matsuda N, Hiromi Y, Miura M. DRONC coordinates cell death and compensatory proliferation. Mol Cellular Biol 2006; 26: 7258-7268.

29. de Nooij JC, Hariharan IK. Uncoupling cell fate determination from patterned cell division in the Drosophila eye. Science (New York, NY) 1995; 270: 983-985.

30. de Nooij JC, Letendre MA, Hariharan IK. A cyclin-dependent kinase inhibitor, Dacapo, is necessary for timely exit from the cell cycle during Drosophila embryogenesis. Cell 1996; 87: 1237-1247.

31. Lane ME, Sauer K, Wallace K, Jan YN, Lehner CF, Vaessin H. Dacapo, a cyclin-dependent kinase inhibitor, stops cell proliferation during Drosophila development. Cell 1996; 87: $1225-1235$

32. Lee CY, Clough EA, Yellon P, Teslovich TM, Stephan DA, Baehrecke EH. Genome-wide analyses of steroid- and radiation-triggered programmed cell death in Drosophila. Curr Biol 2003; 13: 350-357.

33. Zhou L, Steller H. Distinct pathways mediate UV-induced apoptosis in Drosophila embryos. Dev Cell 2003; 4: 599-605.

34. White K, Tahaoglu E, Steller H. Cell killing by the Drosophila gene reaper. Science (New York, NY) 1996; 271: 805-807.

35. Yu SY, Yoo SJ, Yang L, Zapata C, Srinivasan A, Hay BA et al. A pathway of signals regulating effector and initiator caspases in the developing Drosophila eye. Development 2002; 129: 3269-3278.

36. Bourdon JC, Fernandes K, Murray-Zmijewski F, Liu G, Diot A, Xirodimas DP et al. p53 isoforms can regulate p53 transcriptional activity. Genes Dev 2005; 19: 2122-2137.

37. Yang A, Kaghad M, Caput D, McKeon F. On the shoulders of giants: p63, p73 and the rise of p53. Trends Genet 2002; 18: 90-95

38. Ou HD, Lohr F, Vogel V, Mantele W, Dotsch V. Structural evolution of C-terminal domains in the $\mathrm{p} 53$ family. EMBO J 2007; 26: 3463-3473.

39. Janicke RU, Sohn D, Essmann F, Schulze-Osthoff K. The multiple battles fought by anti-apoptotic p21. Cell Cycle 2007; 6: 407-413.

40. Jiang C, Lamblin AF, Steller $H$, Thummel CS. A steroid-triggered transcriptional hierarchy controls salivary gland cell death during Drosophila metamorphosis. Mol Cell 2000; 5: 445-455.

Supplementary Information accompanies the paper on Cell Death and Differentiation website (http://www.nature.com/cdd) 